DOI https://doi.org/10.30525/978-9934-26-019-3-8

\title{
HOMOGENIZATION OF THE MULTI-MODULAR TRANSTROPIC COMPOSITES UNDER TRANSVERSE SHIFT
}

\section{Smoliankova T. M., Klymenko M. I.}

\section{INTRODUCTION}

For the effective usage of composites in engineering and construction, we need to know their mechanical characteristics. They can be different depend on their deformation materials characteristics. In that case we will apply different models of multi-modular theory of elasticity.

In this article we consider concept of the multi-modularity are suggested in the monograph [1]. Author of [2] considers the modification of the multi-modular theory of elasticity. Shift module is considering unchanged in [2]. Experimental data on mechanical characteristics of granular composite are analyzed in publications [3, 4]. Author of [5] considers multi-modular characteristics of isotropic material. Multimodular characteristics of reinforced concrete is viewed in article [6].

Thesis [7] including essence and modern methods of the homogenization of transtropic fibrous composites. Solved problems of the homogenization of transtropic fibrous composites with multi-modular components under transverse and longitudinal stretching and compression are suggested in [8] and [9].

The main purpose of this article is to develop mathematical models of the homogenization of fibrous transversally isotropic composites under deformation of transverse shift.

We develop methods based on kinematic terms of displacement [7] for solving this problem.

\section{Problem statement}

The problem of the homogenization of composites is to determine their mechanical characteristics as a homogenous material for the characteristics of theirs components.

We solve this problem for fibrous transtropic composites. Its components are isotropic matrix and isotropic fiber. Then we determine its effective constants - module of elasticity $E_{2}$ for isotropy plane and Poisson coefficient $v_{23}$. 
Supposing that representative element of composite we can submit as a cell, consists of infinite solid cylinder $(0 \leq r \leq a)$, as a fiber, and infinite hollow cylinder $(a \leq r \leq b)$, as a matrix. Material of matrix and fiber are isotropic and multi-modular.

We consider tasks about transverse (in the plane of isotropy) shift of composite's cell. Modeling this case of deformation of the representative element of composite is based on solving Kirsch's problem [7].

To get a clear shift on outer surface $(r=b)$ of elementary cell we have to apply a load:

$$
\sigma_{r}(b, \theta)=\sigma_{0} \cos 2 \theta, \tau_{r \theta}(b, \theta)=-\sigma_{0} \sin 2 \theta .
$$

In (1) value $\sigma_{0}$ is constant. To get a clear shift we suppose that axial deformations for matrix and fiber are $\varepsilon_{z}=0$. Elasticity module and Poisson coefficient values for multi-modular materials corresponding $E_{+}$and $v_{+}$under stretching and $E_{-}$and $v_{-}$under compression. Symbol is using for material of a matrix and symbol ${ }^{\circ}$ is for material of a fiber.

Ratio between deformation and stress for multi-modular materials according to (1) csn be written as:

$$
\begin{gathered}
\varepsilon_{r}=\frac{1}{E_{+}}\left[\sigma_{r}-v_{+}\left(\sigma_{z}+\sigma_{\theta}\right)\right], \\
\varepsilon_{\theta}=\frac{1}{E_{+}}\left[\sigma_{\theta}-v_{+}\left(\sigma_{r}+\sigma_{z}\right)\right], \\
\varepsilon_{z}=\frac{1}{E_{+}}\left[\sigma_{z}-v_{+}\left(\sigma_{r}+\sigma_{\theta}\right)\right], \\
\gamma_{r \theta}=\frac{2\left(1+v_{+}\right)}{E_{+}} \tau_{r \theta} .
\end{gathered}
$$

On the outer surface $(r=b)$ is a positive radial stress $\sigma_{r}>0$ and axial stress in composites $\sigma_{z}<0$ in ratio (2). We suppose that expression sign of stress $\sigma_{\theta}$ in isotropy plane matches with the sign of stress $\sigma_{r}$. Opposite the signs of stress (2) expressions changed. 


\section{Common deformation of matrix and fiber problem}

We consider the problem of common deformation of matrix and fiber under transverse shift.

Stress components of matrix can be written as [7]:

$$
\begin{gathered}
\sigma_{r}^{*}(r, \theta)=-\left(2 A_{1}+\frac{6 A_{3}}{r^{4}}+\frac{4 A_{4}}{r^{2}}\right) \cos 2 \theta ; \\
\sigma_{\theta}^{*}(r, \theta)=\left(2 A_{1}+12 A_{2} r^{2}+\frac{6 A_{3}}{r^{4}}\right) \cos 2 \theta ; \\
\tau_{r \theta}^{*}(r, \theta)=\left(2 A_{1}+6 A_{2} r^{2}-\frac{6 A_{3}}{r^{4}}-\frac{2 A_{4}}{r^{2}}\right) \sin 2 \theta ; \\
\tau_{z r}^{*}=0 ; \tau_{\theta z}^{*}=0 .
\end{gathered}
$$

Fiber stress taking into their end under $r=0$ can be written as:

$$
\begin{gathered}
\sigma_{r}^{\circ}(r, \theta)=-2 A_{5} \cos 2 \theta ; \\
\sigma_{\theta}^{\circ}(r, \theta)=\left(2 A_{5}+12 A_{6} r^{2}\right) \cos 2 \theta ; \\
\tau_{r \theta}^{\circ}(r, \theta)=\left(2 A_{5}+6 A_{6} r^{2}\right) \sin 2 \theta ; \\
\tau_{z r}^{\circ}=0 ; \tau_{\theta z}^{\circ}=0 .
\end{gathered}
$$

Using condition $\varepsilon_{z}^{*}=0$ we can find components $\sigma_{z}^{*}$.

According to (2), we solve:

$$
\sigma_{z}^{*}=v_{-}^{*}\left(\sigma_{r}^{*}+\sigma_{\theta}^{*}\right)=v_{-}^{*}\left(12 A_{2} r^{2}-\frac{4 A_{4}}{r^{2}}\right) \cos 2 \theta
$$

Similarly according to $\varepsilon_{z}^{\circ}=0$ we find:

$$
\sigma_{z}^{\circ}=v_{-}^{\circ}\left(\sigma_{r}^{\circ}+\sigma_{\theta}^{\circ}\right)=12 A_{6} v_{-}^{\circ} r^{2} \cos 2 \theta
$$

Deformation of matrix can be written as:

$$
\varepsilon_{r}^{*}(r, \theta)=-\frac{1}{E_{+}^{*}}\left(2 A_{1}\left(1+v_{+}^{*}\right)+12 A_{2} r^{2}\left(v_{+}^{*}+v_{+}^{*} v_{-}^{*}\right)+\right.
$$




$$
\begin{gathered}
\left.+\frac{6 A_{1}}{r^{4}}\left(1+v_{+}^{*}\right)+\frac{4 A_{4}}{r^{2}}\left(1-v_{+}^{*} v_{-}^{*}\right)\right) \cos 2 \theta \\
\varepsilon_{\theta}^{*}(r, \theta)=\frac{1}{E_{+}^{*}}\left(2 A_{1}\left(1+v_{+}^{*}\right)+12 A_{2} r^{2}\left(1-v_{+}^{*} v_{-}^{*}\right)+\right. \\
\left.+\frac{6 A_{3}}{r^{4}}\left(1+v_{+}^{*}\right)+\frac{4 A_{4}}{r^{2}}\left(v_{+}^{*}+v_{+}^{*} v_{-}^{*}\right)\right) \cos 2 \theta ; \\
\gamma_{r \theta}^{*}(r, \theta)=\frac{2\left(1+v_{+}^{*}\right)}{E_{+}^{*}}\left(2 A_{1}+6 A_{2} r^{2}-\frac{6 A_{3}}{r^{4}}-\frac{2 A_{4}}{r^{2}}\right) \sin 2 \theta ; \\
\gamma_{z r}^{*}=0 ; \gamma_{z \theta}^{*}=0 .
\end{gathered}
$$

Using (2) and (4) we find deformation of fiber:

$$
\begin{gathered}
\varepsilon_{r}^{\circ}(r, \theta)=-\frac{1}{E_{+}^{\circ}}\left(2 A_{5}\left(1+v_{+}^{\circ}\right)+12 A_{6} r^{2}\left(v_{+}^{\circ}+v_{+}^{\circ} v_{-}^{\circ}\right)\right) \cos 2 \theta \\
\varepsilon_{\theta}^{\circ}(r, \theta)=\frac{1}{E_{+}^{\circ}}\left(2 A_{5}\left(1+v_{+}^{\circ}\right)+12 A_{6} r^{2}\left(1-v_{+}^{\circ} v_{-}^{\circ}\right)\right) \cos 2 \theta ; \\
\gamma_{r \theta}^{\circ}(r, \theta)=\frac{2\left(1+v_{+}^{\circ}\right)}{E_{+}^{\circ}}\left(2 A_{5}+6 A_{6} r^{2}\right) \sin 2 \theta ; \\
\gamma_{z r}^{\circ}=0 ; \gamma_{z \theta}^{\circ}=0 .
\end{gathered}
$$

From the Hooke's law we obtain components of the displacement vector of matrix and fiber.

$$
\begin{gathered}
u_{r}^{*}(r, \theta)=\int \varepsilon_{r}^{*} d r=-\frac{1}{E_{+}^{*}}\left(2 A_{1}\left(1+v_{+}^{*}\right) r+4 A_{2} r^{3}\left(v_{+}^{*}+v_{+}^{*} v_{-}^{*}\right)-\right. \\
\left.-\frac{2 A_{3}}{r^{3}}\left(1+v_{+}^{*}\right)-\frac{4 A_{4}}{r}\left(1-v_{+}^{*} v_{-}^{*}\right)\right) \cos 2 \theta+C_{1}(\theta) . \\
u_{\theta}^{*}(r, \theta)=\int\left(r \varepsilon_{\theta}^{*}-u_{r}^{*}\right) d \theta=\frac{2}{E_{+}^{*}} \times \\
\times\left(A_{1}\left(1+v_{+}^{*}\right) r+A_{2} r^{3}\left(3+v_{+}^{*}-2 v_{+}^{*} v_{-}^{*}\right)+\frac{A_{3}}{r^{3}}\left(1+v_{+}^{*}\right)+\frac{A_{4}}{r}\left(v_{+}^{*}+2 v_{+}^{*} v_{-}^{*}-1\right)\right) \times \\
\times \sin 2 \theta-\int C_{1}(\theta) d \theta+C_{2}(r) .
\end{gathered}
$$

We set up following expressions $u_{r}^{*}(r, \theta)$ and $u_{\theta}^{*}(r, \theta)$ for in Cauchy equation and equate to expressions for $\gamma_{r \theta}^{*}(r, \theta)$ in (7): 


$$
\begin{aligned}
& \frac{2\left(1+v_{+}^{*}\right)}{E_{+}^{*}}\left(2 A_{1}+6 A_{2} r^{2}-\frac{6 A_{3}}{r^{4}}-\frac{2 A_{4}}{r^{2}}\right) \sin 2 \theta= \\
= & \frac{2\left(1+v_{+}^{*}\right)}{E_{+}^{*}}\left(2 A_{1}+6 A_{2} r^{2}-\frac{6 A_{3}}{r^{4}}-\frac{2 A_{4}}{r^{2}}\right) \sin 2 \theta+ \\
& +\frac{1}{r} \frac{\partial C_{1}(\theta)}{\partial \theta}+\frac{\partial C_{2}(r)}{\partial r}+\frac{\jmath C_{1}(\theta) d \theta}{r}-\frac{C_{2}(r)}{r} .
\end{aligned}
$$

We have:

$$
\frac{\partial C_{1}(\theta)}{\partial \theta}+r \frac{\partial C_{2}(r)}{\partial r}+\int C_{1}(\theta) d \theta-C_{2}(r)=0 .
$$

Then we differentiate this equation on $\theta$ :

$$
\frac{\partial^{2} C_{1}(\theta)}{\partial \theta^{2}}+C_{1}(\theta)=0
$$

We obtain (12):

$$
C_{1}(\theta)=H_{1} \cos \theta+H_{2} \sin \theta .
$$

Radial displacements of matrix can be written as:

$$
\begin{gathered}
u_{r}^{*}(r, \theta)=-\frac{1}{E_{+}^{*}}\left(2 A_{1}\left(1+v_{+}^{*}\right) r+4 A_{2} r^{3}\left(v_{+}^{*}+v_{+}^{*} v_{-}^{*}\right)-\right. \\
\left.-\frac{2 A_{3}}{r^{3}}\left(1+v_{+}^{*}\right)-\frac{4 A_{4}}{r}\left(1-v_{+}^{*} v_{-}^{*}\right)\right) \cos 2 \theta+H_{1} \cos \theta+H_{2} \sin \theta .
\end{gathered}
$$

For solving $C_{2}(r)$, we set up (13) in (11):

$$
\begin{gathered}
\frac{\partial\left(H_{1} \cos \theta+H_{2} \sin \theta\right)}{\partial \theta}+r \frac{\partial C_{2}(r)}{\partial r}+\int\left(H_{1} \cos \theta+H_{2} \sin \theta\right) d \theta-C_{2}(r)=0 \\
r \frac{\partial C_{2}(r)}{\partial r}-C_{2}(r)=0
\end{gathered}
$$


Function is a solving of its equation:

$$
C_{2}(r)=H_{3} r
$$

(10) can be written as:

$$
\begin{gathered}
u_{\theta}^{*}(r, \theta)=\frac{2}{E_{+}^{*}}\left(A_{1}\left(1+v_{+}^{*}\right) r+A_{2} r^{3}\left(3+v_{+}^{*}-2 v_{+}^{*} v_{-}^{*}\right)+\frac{A_{3}}{r^{3}}\left(1+v_{+}^{*}\right)+\right. \\
\left.+\frac{A_{4}}{r}\left(v_{+}^{*}+2 v_{+}^{*} v_{-}^{*}-1\right)\right) \sin 2 \theta-H_{1} \sin \theta+H_{2} \cos \theta+H_{3} r .
\end{gathered}
$$

We find constants $H_{1}$ and $H_{2} \mathrm{y}(17)$ according to the symmetry condition of boundary conditions:

$$
H_{1}=0, H_{2}=0, H_{3}=0 .
$$

We have displacements of matrix points as:

$$
\begin{gathered}
u_{r}^{*}(r, \theta)=-\frac{1}{E_{+}^{*}}\left(2 A_{1}\left(1+v_{+}^{*}\right) r+4 A_{2} r^{3}\left(v_{+}^{*}+v_{+}^{*} v_{-}^{*}\right)-\right. \\
\left.-\frac{2 A_{3}}{r^{3}}\left(1+v_{+}^{*}\right)-\frac{4 A_{4}}{r}\left(1-v_{+}^{*} v_{-}^{*}\right)\right) \cos 2 \theta \\
u_{\theta}^{*}(r, \theta)=\frac{2}{E_{+}^{*}}\left(A_{1}\left(1+v_{+}^{*}\right) r+A_{2} r^{3}\left(3+v_{+}^{*}-2 v_{+}^{*} v_{-}^{*}\right)+\right. \\
\left.+\frac{A_{3}}{r^{3}}\left(1+v_{+}^{*}\right)+\frac{A_{4}}{r}\left(v_{+}^{*}+2 v_{+}^{*} v_{-}^{*}-1\right)\right) \sin 2 \theta
\end{gathered}
$$

According to Cauchy equation, we obtain components of displacement vector for fiber.

$$
\begin{gathered}
u_{r}^{\circ}(r, \theta)=\int \varepsilon_{r r}^{\circ} d r=-\frac{1}{E_{+}^{\circ}}\left(2 A_{5}\left(1+v_{+}^{\circ}\right) r+4 A_{6} r^{3}\left(v_{+}^{\circ}+v_{+}^{\circ} v_{-}^{\circ}\right)\right) \cos 2 \theta+T_{1}(\theta) . \\
u_{\theta}^{\circ}(r, \theta)=\int\left(r \varepsilon_{\theta}^{\circ}-u_{r}^{\circ}\right) d \theta=\frac{2}{E_{+}^{\circ}} \times \\
\times\left(A_{5}\left(1+v_{+}^{\circ}\right) r+A_{6} r^{3}\left(3+v_{+}^{\circ}-2 v_{+}^{\circ} v_{-}^{\circ}\right)\right) \sin 2 \theta-\int T_{1}(\theta) d \theta+T_{2}(r) .
\end{gathered}
$$


We set up following expressions for $u_{r}^{\circ}(r, \theta)$ and $u_{\theta}^{\circ}(r, \theta)$ into Cauchy equation and equate to expressions for $\gamma_{r \theta}^{\circ}(r, \theta)$ in (8):

$$
\begin{gathered}
\frac{2\left(1+v_{+}^{\circ}\right)}{E_{+}^{\circ}}\left(2 A_{5}+6 A_{6} r^{2}\right) \sin 2 \theta=\frac{2\left(1+v_{+}^{\circ}\right)}{E_{+}^{\circ}}\left(2 A_{5}+6 A_{6} r^{2}\right) \sin 2 \theta+ \\
+\frac{1}{r} \frac{\partial T_{1}(\theta)}{\partial \theta}+\frac{\partial T_{2}(r)}{\partial r}+\frac{\mathrm{J} T_{1}(\theta) d \theta}{r}-\frac{T_{2}(r)}{r} .
\end{gathered}
$$

We obtain:

$$
\frac{\partial T_{1}(\theta)}{\partial \theta}+r \frac{\partial T_{2}(r)}{\partial r}+\int T_{1}(\theta) d \theta-T_{2}(r)=0 .
$$

Similarly (11) we have:

$$
T_{1}(\theta)=R_{1} \cos \theta+R_{2} \sin \theta .
$$

Radial displacements of fiber can be written as:

$$
\begin{gathered}
u_{r}^{\circ}(r, \theta)=-\frac{1}{E_{+}^{\circ}}\left(2 A_{5}\left(1+v_{+}^{\circ}\right) r+4 A_{6} r^{3}\left(v_{+}^{\circ}+v_{+}^{\circ} v_{-}^{\circ}\right)\right) \cos 2 \theta+ \\
+R_{1} \cos \theta+R_{2} \sin \theta .
\end{gathered}
$$

Because $u_{r}^{\circ}(0, \theta)=0$, for arbitrary $\theta$ we have:

$$
R_{1} \cos \theta+R_{2} \sin \theta=0 \text {. }
$$

So, $R_{1}=0, R_{2}=0$.

For determining $T_{2}(r)$ from (22) we obtain the ratio:

$$
r \frac{\partial T_{2}(r)}{\partial r}-T_{2}(r)=0
$$

From (26) we solve: 


$$
T_{2}(r)=R_{3} r
$$

From symmetry of boundary conditions we have $R_{3}=0$.

$$
\begin{gathered}
u_{r}^{\circ}(r, \theta)=-\frac{1}{E_{+}^{\circ}}\left(2 A_{5}\left(1+v_{+}^{\circ}\right) r+4 A_{6} r^{3}\left(v_{+}^{\circ}+v_{+}^{\circ} v_{-}^{\circ}\right)\right) \cos 2 \theta \\
u_{\theta}^{\circ}(r, \theta)=\frac{2}{E_{+}^{\circ}}\left(A_{5}\left(1+v_{+}^{\circ}\right) r+A_{6} r^{3}\left(3+v_{+}^{\circ}-2 v_{+}^{\circ} v_{-}^{\circ}\right)\right) \sin 2 \theta .
\end{gathered}
$$

We solve problem of common transverse shift of a solid cylinder as a fiber and hollow cylinder as a matrix. So we have to find unknown constants $A_{i}, i=1, \ldots, 6$ included in components of displacements and stresses of matrix and fiber. We use boundary conditions (1) on outer surface of a cell and conditions of continuity for displacements and stresses under $r=a$ :

$$
\begin{aligned}
& u_{r}^{*}(a, \theta)=u_{r}^{\circ}(a, \theta), \\
& \sigma_{r}^{*}(a, \theta)=\sigma_{r}^{\circ}(a, \theta), \\
& u_{\theta}^{*}(a, \theta)=u_{\theta}^{\circ}(a, \theta), \\
& \tau_{r \theta}^{*}(a, \theta)=\tau_{r \theta}^{\circ}(a, \theta) .
\end{aligned}
$$

We obtain:

$$
\sigma_{0}=-2 A_{1}-\frac{6 A_{3}}{b^{4}}-\frac{4 A_{4}}{b^{2}} ;-\sigma_{0}=2 A_{1}+6 A_{2} b^{2}-\frac{6 A_{3}}{b^{4}}-\frac{2 A_{4}}{b^{2}},
$$

or

$$
A_{3}=\frac{A_{2} b^{6}}{2}-\frac{A_{4} b^{2}}{2} ; A_{1}=-\frac{\sigma_{0}}{2}-\frac{3 A_{2} b^{2}}{2}-\frac{A_{4}}{2 b^{2}} .
$$

According to the continuity of stresses (30), (32), we obtain:

$$
A_{5}=-\frac{\sigma_{0}}{2}+\frac{3 A_{2} b^{2}\left(b^{4}-a^{4}\right)}{2 a^{4}}+\frac{A_{4}\left(4 a^{2} b^{2}-a^{4}-3 b^{4}\right)}{2 a^{4} b^{2}},
$$




$$
A_{6}=\frac{A_{2}\left(a^{6}-b^{6}\right)}{a^{6}}+\frac{A_{4}\left(b^{2}-a^{2}\right)}{a^{6}} .
$$

From (29) and (31), we have:

$$
\begin{gathered}
\frac{1}{E_{+}^{*}}\left(2 A_{1}\left(1+v_{+}^{*}\right) a+4 A_{2} a^{3}\left(v_{+}^{*}+v_{+}^{*} v_{-}^{*}\right)-\right. \\
\left.-\frac{2 A_{3}}{a^{3}}\left(1+v_{+}^{*}\right)-\frac{4 A_{4}}{a}\left(1-v_{+}^{*} v_{-}^{*}\right)\right)=\frac{1}{E_{+}^{\circ}}\left(2 A_{5}\left(1+v_{+}^{\circ}\right) a+4 A_{6} a^{3}\left(v_{+}^{\circ}+v_{+}^{\circ} v_{-}^{\circ}\right)\right) \\
\frac{1}{E_{+}^{*}}\left(A_{1}\left(1+v_{+}^{*}\right) a+A_{2} a^{3}\left(3+v_{+}^{*}-2 v_{+}^{*} v_{-}^{*}\right)+\frac{A_{3}}{a^{3}}\left(1+v_{+}^{*}\right)+\right. \\
\left.+\frac{A_{4}}{a}\left(v_{+}^{*}+2 v_{+}^{*} v_{-}^{*}-1\right)\right)=\frac{1}{E_{+}^{\circ}}\left(A_{5}\left(1+v_{+}^{\circ}\right) a+A_{6} a^{3}\left(3+v_{+}^{\circ}-2 v_{+}^{\circ} v_{-}^{\circ}\right)\right)
\end{gathered}
$$

Using Eq. (33) the following equations can be written as system of two of them:

$$
\left\{\begin{array}{l}
d_{11} b^{2} B+\frac{d_{12}}{a^{2}} D=\sigma_{0} b_{1}, \\
d_{21} b^{2} B+\frac{d_{22}}{a^{2}} D=\sigma_{0} b_{1} .
\end{array}\right.
$$

Introduce the notation:

$$
\begin{gathered}
d_{11}=E_{+}^{\circ}\left(\left(4 f-3-\frac{1}{f^{2}}\right)\left(1+v_{+}^{*}\right)-4 f\left(1-v_{+}^{*} v_{-}^{*}\right)\right)- \\
-E_{+}^{*}\left(\left(4 f-\frac{1}{f^{2}}-3\right)\left(1+v_{+}^{\circ}\right)+4\left(\frac{1}{f^{2}}-f\right)\left(1-v_{+}^{\circ} v_{-}^{\circ}\right)\right) ; \\
d_{12}=E_{+}^{\circ}\left(\left(\frac{1}{f}-f\right)\left(1+v_{+}^{*}\right)-4\left(1-v_{+}^{*} v_{-}^{*}\right)\right)- \\
-E_{+}^{*}\left(\left(\frac{1}{f}-f\right)\left(1+v_{+}^{\circ}\right)+4\left(1-\frac{1}{f}\right)\left(1-v_{+}^{\circ} v_{-}^{\circ}\right)\right) ; \\
d_{21}=E_{+}^{\circ}\left(4 f\left(1-v_{+}^{*} v_{-}^{*}\right)+\left(2 f+\frac{1}{f^{2}}-3\right)\left(1+v_{+}^{*}\right)\right)- \\
-E_{+}^{*}\left(\left(2 f+\frac{1}{f^{2}}-3\right)\left(1+v_{+}^{\circ}\right)+4\left(f-\frac{1}{f^{2}}\right)\left(1-v_{+}^{\circ} v_{-}^{\circ}\right)\right) ; \\
d_{22}=E_{+}^{\circ}\left(\left(2-f-\frac{1}{f}\right)\left(1+v_{+}^{*}\right)-4\left(1-v_{+}^{*} v_{-}^{*}\right)\right)-
\end{gathered}
$$




$$
\begin{gathered}
-E_{+}^{*}\left(\left(2-f-\frac{1}{f}\right)\left(1+v_{+}^{\circ}\right)+4\left(\frac{1}{f}-1\right)\left(1-v_{+}^{\circ} v_{-}^{\circ}\right)\right) ; \\
b_{1}=E_{+}^{\circ}\left(1+v_{+}^{*}\right)-E_{+}^{*}\left(1+v_{+}^{\circ}\right) .
\end{gathered}
$$

According to (37), we have:

$$
\begin{gathered}
A_{2}=\frac{\sigma_{0} b_{1}}{b^{2}} \frac{d_{22}-d_{12}}{d_{11} d_{22}-d_{21} d_{12}}, \\
A_{4}=\sigma_{0} a^{2} b_{1} \frac{d_{11}-d_{21}}{d_{11} d_{22}-d_{21} d_{12}}
\end{gathered}
$$

Then we obtain other undefined constants:

$$
\begin{gathered}
A_{5}=\frac{\sigma_{0}}{2}\left(\frac{b_{1}}{d_{11} d_{22}-d_{21} d_{12}}\left(3\left(\frac{1}{f^{2}}-1\right)\left(d_{22}-d_{12}\right)+\left(4-f-\frac{3}{f}\right)\left(d_{11}-d_{21}\right)\right)-1\right) \\
A_{6}=\frac{\sigma_{0} b_{1}}{a^{2}\left(d_{11} d_{22}-d_{21} d_{12}\right)}\left(\left(f-\frac{1}{f^{2}}\right)\left(d_{22}-d_{12}\right)+\left(\frac{1}{f}-1\right)\left(d_{11}-d_{21}\right)\right) \\
A_{3}=\frac{\sigma_{0} b_{1} b^{4}}{2\left(d_{11} d_{22}-d_{21} d_{12}\right)}\left(\left(d_{22}-d_{12}\right)-f\left(d_{11}-d_{21}\right)\right) ; \\
A_{1}=-\frac{\sigma_{0}}{2}\left(1+\frac{b_{1}}{d_{11} d_{22}-d_{21} d_{12}}\left(3\left(d_{22}-d_{12}\right)+f\left(d_{11}-d_{21}\right)\right)\right)
\end{gathered}
$$

The stress of the matrix can be written as:

$$
\begin{gathered}
\sigma_{r}^{*}(r, \theta)=\sigma_{0}\left(1+\frac{b_{1}}{d_{11} d_{22}-d_{21} d_{12}}\left(3\left(1-\frac{b^{4}}{r^{4}}\right)\left(d_{22}-d_{12}\right)+\right.\right. \\
\left.\left.+f\left(1+\frac{3 b^{4}}{r^{4}}-\frac{4 b^{2}}{r^{2}}\right)\left(d_{11}-d_{21}\right)\right)\right) \cos 2 \theta ; \\
\quad \sigma_{\theta}^{*}(r, \theta)=-\sigma_{0}\left(1+\frac{b_{1}}{d_{11} d_{22}-d_{21} d_{12}} \times\right. \\
\left.\times\left(3\left(1-4 \frac{r^{2}}{b^{2}}-\frac{b^{4}}{r^{4}}\right)\left(d_{22}-d_{12}\right)+f\left(1+\frac{3 b^{4}}{r^{4}}\right)\left(d_{11}-d_{21}\right)\right)\right) \cos 2 \theta ; \\
\tau_{r \theta}^{*}(r, \theta)=-\sigma_{0}\left(1+\frac{b_{1}}{d_{11} d_{22}-d_{21} d_{12}}\left(3\left(1-\frac{2 r^{2}}{b^{2}}+\frac{b^{4}}{r^{4}}\right)\left(d_{22}-d_{12}\right)+\right.\right.
\end{gathered}
$$




$$
\begin{gathered}
\left.\left.+f\left(d_{11}-d_{21}\right)\left(1-\frac{3 b^{4}}{r^{4}}+\frac{2 b^{2}}{r^{2}}\right)\right)\right) \sin 2 \theta \\
\sigma_{z}^{*}(r, \theta)=\frac{4 \sigma_{0} v_{12}^{*} b_{1}}{r^{2} b^{2}\left(d_{11} d_{22}-d_{21} d_{12}\right)}\left(3 r^{4}\left(d_{22}-d_{12}\right)-a^{2} b^{2}\left(d_{11}-d_{21}\right)\right) \cos 2 \theta .
\end{gathered}
$$

For the displacements of the matrix we obtain:

$$
\begin{gathered}
u_{r}^{*}(r, \theta)=\frac{\sigma_{0} r}{E_{+}^{*}}\left(\left(1+v_{+}^{*}\right)+\frac{b_{1}}{\left(d_{11} d_{22}-d_{21} d_{12}\right)} \times\right. \\
\times\left(\left(\left(3+\frac{b^{4}}{r^{4}}\right)\left(1+v_{+}^{*}\right)-\frac{4 r^{2}}{b^{2}}\left(v_{+}^{*}+v_{+}^{*} v_{-}^{*}\right)\right)\left(d_{22}-d_{12}\right)+\right. \\
\left.\left.+\left(\frac{4 a^{2}}{r^{2}}\left(1-v_{+}^{*} v_{-}^{*}\right)+f\left(1+v_{+}^{*}\right)\left(1-\frac{b^{4}}{r^{4}}\right)\right)\left(d_{11}-d_{21}\right)\right)\right) \cos 2 \theta \\
u_{\theta}^{*}(r, \theta)=\frac{\sigma_{0} r}{E_{+}^{*}}\left(-\left(1+v_{+}^{*}\right)+\frac{b_{1}}{\left(d_{11} d_{22}-d_{21} d_{12}\right)} \times\right. \\
\times\left(\left(\frac{2 r^{2}}{b^{2}}\left(3+v_{+}^{*}-2 v_{+}^{*} v_{-}^{*}\right)+\left(\frac{b^{4}}{r^{4}}-3\right)\left(1+v_{+}^{*}\right)\right)\left(d_{22}-d_{12}\right)+\right. \\
\left.\left.+\left(\frac{2 a^{2}}{r^{2}}\left(v_{+}^{*}+2 v_{+}^{*} v_{-}^{*}-1\right)-\left(1+v_{+}^{*}\right) f\left(1+\frac{b^{4}}{r^{4}}\right)\right)\left(d_{11}-d_{21}\right)\right)\right) \sin 2 \theta .
\end{gathered}
$$

We have following expressions of stress of fiber's points:

$$
\begin{gathered}
\sigma_{r}^{\circ}(r, \theta)=\sigma_{0}\left(1-\frac{b_{1}}{d_{11} d_{22}-d_{21} d_{12}}\left(3\left(\frac{1}{f^{2}}-1\right)\left(d_{22}-d_{12}\right)+\right.\right. \\
\left.\left.+\left(4-f-\frac{3}{f}\right)\left(d_{11}-d_{21}\right)\right)\right) \cos 2 \theta ; \\
\sigma_{\theta}^{\circ}(r, \theta)=\sigma_{0}\left(\frac { b _ { 1 } } { ( d _ { 1 1 } d _ { 2 2 } - d _ { 2 1 } d _ { 1 2 } ) } \left(3\left(\left(1-\frac{4 r^{2}}{a^{2}}\right) \frac{1}{f^{2}}-1+\frac{4 r^{2}}{a^{2}} f\right)\left(d_{22}-d_{12}\right)+\right.\right. \\
\left.\left.+\left(4\left(1-\frac{3 r^{2}}{a^{2}}\right)-f+\frac{3}{f}\left(\frac{4 r^{2}}{a^{2}}-1\right)\right)\left(d_{11}-d_{21}\right)\right)-1\right) \cos 2 \theta ; \\
\tau_{r \theta}^{\circ}(r, \theta)=\sigma_{0}\left(\frac { b _ { 1 } } { ( d _ { 1 1 } d _ { 2 2 } - d _ { 2 1 } d _ { 1 2 } ) } \left(3\left(\frac{1}{f^{2}}\left(1-\frac{2 r^{2}}{a^{2}}\right)-1+\frac{2 r^{2}}{a^{2}} f\right)\left(d_{22}-d_{12}\right)+\right.\right.
\end{gathered}
$$




$$
\begin{gathered}
\left.\left.+\left(2\left(2-\frac{3 r^{2}}{a^{2}}\right)-f+\frac{3}{f}\left(\frac{2 r^{2}}{a^{2}}-1\right)\right)\left(d_{11}-d_{21}\right)\right)-1\right) \sin 2 \theta \\
\sigma_{z}^{\circ}(r, \theta)=\frac{12 \sigma_{0} b_{1}}{\left(d_{11} d_{22}-d_{21} d_{12}\right)} \frac{r^{2}}{a^{2}}\left(\left(f-\frac{1}{f^{2}}\right)\left(d_{22}-d_{12}\right)+\left(\frac{1}{f}-1\right)\left(d_{11}-d_{21}\right)\right) \times \\
\times v_{12}^{\circ} \cos 2 \theta .
\end{gathered}
$$

Displacements of fiber can be written as:

$$
\begin{gathered}
u_{r}^{\circ}(r, \theta)=\frac{\sigma_{0} r}{E_{+}^{\circ}}\left(\left(1+v_{+}^{\circ}\right)-\frac{b_{1}}{\left(d_{11} d_{22}-d_{21} d_{12}\right)} \times\right. \\
\times\left(\left(\left(\frac{3}{f^{2}}-3\right)\left(1+v_{+}^{\circ}\right)+\frac{4 r^{2}}{a^{2}}\left(f-\frac{1}{f^{2}}\right)\left(v_{+}^{\circ}+v_{+}^{\circ} v_{-}^{\circ}\right)\right)\left(d_{22}-d_{12}\right)+\right. \\
\left.\left.+\left(\left(4-f-\frac{3}{f}\right)\left(1+v_{+}^{\circ}\right)+\frac{4 r^{2}}{a^{2}}\left(\frac{1}{f}-1\right)\left(v_{+}^{\circ}+v_{+}^{\circ} v_{-}^{\circ}\right)\right)\left(d_{11}-d_{21}\right)\right)\right) \cos 2 \theta \\
u_{\theta}^{\circ}(r, \theta)=\frac{\sigma_{0} r}{E_{+}^{\circ}}\left(-\left(1+v_{+}^{\circ}\right)+\frac{b_{1}}{\left(d_{11} d_{22}-d_{21} d_{12}\right)} \times\right. \\
\left(\left(\left(\frac{3}{f^{2}}-3\right)\left(1+v_{+}^{\circ}\right)+\frac{2 r^{2}}{a^{2}}\left(f-\frac{1}{f^{2}}\right)\left(3+v_{+}^{\circ}-2 v_{+}^{\circ} v_{-}^{\circ}\right)\right)\left(d_{22}-d_{12}\right)+\right. \\
\left.\left.+\left(\left(4-f-\frac{3}{f}\right)\left(1+v_{+}^{\circ}\right)+\frac{2 r^{2}}{a^{2}}\left(\frac{1}{f}-1\right)\left(3+v_{+}^{\circ}-2 v_{+}^{\circ} v_{-}^{\circ}\right)\right)\left(d_{11}-d_{21}\right)\right)\right) \sin 2 \theta .
\end{gathered}
$$

We solve similarly problem of clear transverse shift of transtropic homogeneous material as a composite. In that case composite materials considered as a solid cylinder with radius $b$. The boundary conditions can be written as (1). Components of stress-strain state of a homogeneous modeling similarly to fiber, so we have:

$$
\begin{gathered}
\sigma_{r}(r, \theta)=-2 \tilde{A}_{1} \cos 2 \theta, \\
\tau_{r \theta}(r, \theta)=\left(2 \tilde{A}_{1}+6 \tilde{A}_{2} r^{2}\right) \sin 2 \theta .
\end{gathered}
$$

According to boundary conditions, we obtain: 


$$
\begin{gathered}
\tilde{A}_{1}=-\frac{\sigma_{0}}{2} . \\
\tilde{A}_{2}=0 .
\end{gathered}
$$

Using ratios for the fiber and matrix and (46), (47) for the homogeneous transtropic material which modeled composite as follows:

$$
\begin{gathered}
\sigma_{r}(r, \theta)=\sigma_{0} \cos 2 \theta ; \sigma_{\theta}(r, \theta)=-\sigma_{0} \cos 2 \theta ; \\
\tau_{r \theta}(r, \theta)=-\sigma_{0} \sin 2 \theta ; \\
\varepsilon_{r}(r, \theta)=\frac{\sigma_{0}\left(1+v_{23}^{+}\right)}{E_{2}^{+}} \cos 2 \theta ; \varepsilon_{\theta}(r, \theta)=-\frac{\sigma_{0}\left(1+v_{23}^{+}\right)}{E_{2}^{+}} \cos 2 \theta ; \\
\gamma_{r \theta}(r, \theta)=-\frac{2 \sigma_{0}\left(1+v_{23}^{+}\right)}{E_{2}^{+}} \sin 2 \theta ; \\
u_{r}(r, \theta)=\frac{\sigma_{0}\left(1+v_{23}^{+}\right) r}{E_{2}^{+}} \cos 2 \theta, \\
u_{\theta}(r, \theta)=-\frac{\sigma_{0}\left(1+v_{23}^{+}\right) r}{E_{2}^{+}} \sin 2 \theta .
\end{gathered}
$$

According to the conditions of harmonization - equality of radial displacements of homogeneous composite and radial displacements of matrix on an outer border of a composite cell, we obtain effective elastic constants of a homogeneous composite:

$$
u_{r}(b, \theta)=u_{r}^{*}(b, \theta) .
$$

Using Eq. (51), we have:

$$
\begin{gathered}
\frac{\left(1+v_{23}^{+}\right)}{E_{2}^{+}}=\frac{1}{E_{+}^{*}}\left(\left(1+v_{+}^{*}\right)+\frac{b_{1}}{d_{11} d_{22}-d_{21} d_{12}}\left(\left(3\left(d_{22}-d_{12}\right)+f\left(d_{11}-d_{21}\right)\right) \times\right.\right. \\
\times\left(1+v_{+}^{*}\right)-4\left(d_{22}-d_{12}\right)\left(v_{+}^{*}+v_{+}^{*} v_{-}^{*}\right)+\left(\left(d_{22}-d_{12}\right)-f\left(d_{11}-d_{21}\right)\right) \times \\
\left.\left.\times\left(1+v_{+}^{*}\right)+4 f\left(d_{11}-d_{21}\right)\left(1-v_{+}^{*} v_{-}^{*}\right)\right)\right) .
\end{gathered}
$$

Introduce the notation: 


$$
\chi_{1}=\frac{\left(d_{11}-d_{21}\right)}{d_{11} d_{22}-d_{21} d_{12}}, \chi_{2}=\frac{\left(d_{22}-d_{12}\right)}{d_{11} d_{22}-d_{21} d_{12}} .
$$

After transformations, we expressed:

$$
\frac{1+v_{23}^{+}}{E_{2}^{+}}=\frac{1}{E_{+}^{*}}\left(\left(1+v_{+}^{*}\right)+4\left(\gamma-2 E_{+}^{*}\right)\left(1-v_{+}^{*} v_{-}^{*}\right)\left(f \chi_{1}+\chi_{2}\right)\right) .
$$

In article [7] we give the following ratio $\frac{1-v_{23}^{+}}{E_{2}^{+}}$. According this ratio, (54) and using following condition $\frac{1-v_{23}}{E_{2}}+\frac{1+v_{23}}{E_{2}}=\frac{2}{E_{2}}$, we find:

$$
E_{2}^{+}=\frac{2 \alpha E_{+}^{*}}{\alpha\left(\left(1+v_{+}^{*}\right)+4\left(\gamma-2 E_{+}^{*}\right)\left(1-v_{+}^{*} v_{-}^{*}\right)\left(f \chi_{1}+\chi_{2}\right)\right)+\delta+\eta_{1} \eta_{2}} .
$$

Introduce the notation:

$$
\begin{gathered}
\alpha=E_{+}^{*}(1-f)\left(1-v_{+}^{\circ}\right)+E_{+}^{\circ}\left(f\left(1-v_{+}^{*}\right)+\left(1+v_{+}^{*}\right)\right), \\
\delta=f\left(1+v_{+}^{*}\right)\left(\gamma-2 E_{+}^{\circ}\right)+\gamma\left(1-v_{+}^{*}\right), \\
\eta_{1}=2 E_{+}^{\circ} E_{+}^{*} f(f-1)\left(v_{+}^{*} \cdot \gamma-2 v_{+}^{\circ} E_{+}^{*}\right)^{2}, \\
\eta_{2}=1 /\left(E_{+}^{*}\left(\alpha\left(E_{+}^{\circ} f+E_{+}^{*}(1-f)\right)-2 \beta\left(v_{+}^{*} E_{+}^{\circ} f+v_{12}^{\circ} E_{+}^{*}(1-f)\right)\right)\right), \\
\gamma=E_{+}^{*}\left(1-v_{+}^{\circ}\right)+E_{2}^{\circ}\left(1+v_{+}^{*}\right) .
\end{gathered}
$$

Using ratio $\frac{1+v_{23}}{E_{2}}-\frac{1-v_{23}}{E_{2}}=\frac{2 v_{23}}{E_{2}},(54),(55)$, we obtain expression for Poisson coefficient $v_{23}$ :

$$
v_{23}^{+}=\frac{\alpha\left(\left(1+v_{+}^{*}\right)+4\left(\gamma-2 E_{+}^{*}\right)\left(1-v_{+}^{*} v_{-}^{*}\right)\left(f \chi_{1}+\chi_{2}\right)\right)-\left(\delta+\eta_{1} \eta_{2}\right)}{\alpha\left(\left(1+v_{+}^{*}\right)+4\left(\gamma-2 E_{+}^{*}\right)\left(1-v_{-}^{*} v_{+}^{*}\right)\left(f \chi_{1}+\chi_{2}\right)\right)+\left(\delta+\eta_{1} \eta_{2}\right)} .
$$

In case of transverse shift caused by transverse compression and longitudinal stretching homogeneous mechanical characteristics can be 
solved with ratios (55), (56), where we have to take opposite sings in index.

\section{CONCLUSIONS}

To design and construct materials made of composites we have to know effective elastic constants of its materials solved with considering of differences between their mechanical characteristics under compression and stretching deformation. We can find homogenization of a composite using kinematic terms of harmonization.

Effective values of transverse module of elasticity $E_{2}$ and Poisson coefficient for isotropy plane of transversally isotropic composite can be solved according to common transverse shift of matrix and fiber and similarly problem for deformation of homogeneous composite. Equations of radial displacements on outer surface and common composite's cell are the kinematic terms of harmonization.

Following effective elastic constants can be used to design and construct materials made of multi-modular composites.

\section{SUMMARY}

In this thesis we solve the problem of homogenization of multimodular transtropic composite under transverse shift. It's consists of isotropic elastic matrix and fiber. Mechanical characteristics of composite and its components under compression and stretching are different. In this article we solved effective module of elasticity and Poisson coefficient for isotropy plane of transtropic composite. So we use kinematic terms of harmonization of radial displacements on outer surface and common composite's cell.

\section{REFERENCES}

1. S.A. Ambartsumian, Theory of different modules elasticity, Moscow, Nauka, 1982.

2. I.Y. Tsvelodub, About multi-modular theory of elasticity, Applied mathematics and theoretical physics. Vol. 49, № 1 (2008), 157-164.

3. A.U. Yershova, M.I. Martirosov, Experimental study heterogeneous polymer composites with finely divided filler, Moscow Aviation Institute (National Research University). № 5 (2015), 68-72.

4. D.E. Bessonov, Yu.P. Zezin, E.V. Lomakin, Multimodulus Behevior of the Grained Composites on the Base of Unsaturated Polyetheres, News of Saratov University. Vol. 9 (2009), 9-13. 
5. Pahomov B.M., Alternative Model of Isotropic Material with Different Modulus, Bulletin of MSTU im. Ad Bauman. № 6 (2017), 35-45.

6. Nassef A.S.E., Dahim M.A. New Bi-modular Material Approach to Buckling Problem of Reinforced Concrete Columns. Mechanical EngineeringResearch. 2016. Vol. 6. № 1. P. 19-28.

7. Grebenyuk S., Homeniuk S., Klymenko M. Napruzhenodeformovanyi stan prostorovykh konstruktsii na osnovi homohenizatsii voloknystykh kompozytiv: monohrafiia. Kherson: Vydavnychyi dim «Helvetyka», 2019.

8. Grebenyuk S., Klymenko M., Smoliankova T., Koval R. Effective Characteristics of the Multi-Modular Composites under Transverse Stretching. Actual Problems of Engineering Mechanics, Materials Science Forum.- Trans Tech Publication Ltd, 2019. Vol. 968. P. 511-518.

9. Grebenyuk S., Smoliankova T., Klymenko M., Kudin O. The homogenization of multi-modular composites at their longitudinal deformation. Eastern European Journal of enterprise technologies, 2020. Vol. 3/7 (105).

\section{Information about the authors:} Smoliankova T. M.,

Post Graduate Student at the Department of Fundamental Mathematics, Zaporizhzhia National University 66, Zhukovskoho str., Zaporizhzhia, 69600, Ukraine

Klymenko M. I., $\mathrm{PhD}$, Associate Professor at the Department of Fundamental Mathematics, Zaporizhzhia National University 66, Zhukovskoho str., Zaporizhzhia, 69600, Ukraine 